\title{
Learning to Drive with and without Intelligent Computer Systems and Sensors to Assist
}

\author{
David Adrian Sanders, Giles Eric Tewkesbury, Hassan Parchizadeh, Josh Robertson, Peter Osagie Omoarebun, \\ Manish Malik \\ School of Engineering \\ University of Portsmouth, Portsmouth, UK \\ \{david.sanders, alexander.gegov, hassan.parchizadeh, josh.robertson, Peter.Omoarebun1, manish.malik\}@port.ac.uk
}

\begin{abstract}
This paper investigates whether using sensors during training is more effective than not. Results are presented from investigating novice vehicle drivers learning while using varying amounts of sensor support. Qualitative and quantitative data evaluations are made to compare drivers with sensors against those without while they learn to drive a vehicle. Reliance on the teaching processes used was recorded while various amounts of support were given by the intelligent systems. The work considers whether skilled drivers trained with sensors assisting them during training, could then work well without any assistance from sensors. Finally, some results are included. In all situations, assistance becomes more useful as environments became more complicated.
\end{abstract}

Keywords—Learning; driving; vehicle; assistive; ultrasonic; sensor

\section{INTRODUCTION}

The way that a vehicle driver adapts their actions when presented with various distinct levels of support from a sensor system is explored in this study. The consequences of giving some assistance to drivers, as they learn how to drive, is evaluated for both skilled and novice drivers.

Advanced driver-assistance systems automate, adapt and enhance vehicle systems to improve driving and safety. Safety features can avoid collision or accidents. Technology can be used to avoid collisions and/or alert drivers about difficulties and can take over all or some control of a vehicle. Features can include adaptive cruise control and automatic braking or can alert a driver about other cars on the road or about potential danger. They can keep a vehicle in the correct lane or consider and/or reveal anything in a blind spot. In emergencies, efficient interaction between the vehicle and a human driver can make all the difference. This research explores that collaboration and interfacing [1]-[8].

Several driver-assistance systems features are being built in to modern cars and others can be available as optional add-on packages or as after-market solutions [9]. Driver-assistance systems rely on inputs from sensors such as radar, imaging and vision, in-car networking and Light Detection and Ranging (LiDAR).

Driver-assistance is fast growing [10], [11] with increasing adoption rates and specific standards being developed. For example, communications protocols such as Vehicle Information API [12] or IEEE 2020 for image quality.
In addition, vehicle-to-infrastructure and vehicle-to-vehicle systems can be added, for example Wi-Fi, mobile telephony or data networks [13].

The appropriate level of automated assistance depends on several factors. This research explored the way that vehicle drivers should be trained based on varying levels of advanced assistance available. Research had suggested that an experienced driver trained to drive a vehicle without that assistance from sensors can complete tasks more efficiently when sensors were utilised to assist them [14]-[16]. This paper considers whether skilled drivers trained with sensors assisting them during training, could then work well without any assistance from sensors. Changes in participant behaviour were scrutinised while they were provided with different levels of assistance from sensors. This research shows that they altered their behaviour in new circumstances and environments.

In traditional research, drivers have usually been expert drivers using varying levels of assistance. The acquiring of driving skill has usually been ignored [17]. The research presented in this paper explores drivers in a simple vehicle fitted with sensors as a realistic example of humans interacting with computers. Results from experiments are presented.

Similar studies that used industrial robots were presented in [18], [19]. In the research described in this paper, constraintbased support was used with virtual force-feedback [17]. The closeness to an obstacle (for example a wall) was represented by force feedback and that was akin to research described by Volpe [20] who represented the force felt by a slave robot arm at a remote joystick. The force back in the controller was used to take action to evade collision and to turn a vehicle to head in a safer direction.

The vehicle system is described in [16]. Section II of this paper describes the experiments and presents some results. Section III is a discussion and conclusions from the results obtained.

\section{EXPERIMENTATION}

Drivers used a joystick to control their vehicle. A choice of three different amounts of support could be provided to a vehicle driver:

Setting 0: Sensors were turned off so that there wasn't any force-feedback. Vehicle drivers could drive the vehicle without interference from any sensors. Drivers had the greatest 
autonomy with Setting 0 but the likelihood of crashing was greatest.

Setting 1: Sensors were engaged and the joystick received force-feedback when the vehicle was close to obstacles. As distance to an object reduced, the size of repelling force increased.

Setting 2: Sensors were engaged and the vehicle automatically steered away from objects to avoid collisions. A driver did not sense any resistive force if the vehicle moved in the direction of an obstacles but the vehicle was safely steered.

Setting 3: In Setting 3, both Setting 1 and Setting 2 were delivered. Drivers were the most constrained in this case.

If a driver applied more effort to their joystick then they could always make their vehicle move in opposition to any deflecting force.

Performance measures were the average completion time and number of collisions.

\section{A. Initial Tests}

Initial tests compared learning in Setting 0 (sensors switched off) and Setting 3 (sensors switched on and automatic avoidance safely steering the vehicle around obstacles). Subsequent tests compared learning at Setting 0, Setting 1 and Setting 2 when driving along different courses in different environments.

Drivers drove a Bobcat II vehicle [16] through one of four different courses. The courses were different lengths and contained different numbers of obstacles. Drivers used a Force feedback Pro joystick connected to the vehicle to drive through each course while avoiding obstacles. It had been designed for games and had force-feedback built in and had been used successfully during experiments at Tohoku University [17] and so was chosen for this work

For Setting 3 tests, the resistive force grew bigger when a participant drove their vehicle closer to an obstacle. So that the assistance provided was more restricted than Setting 1 or Setting 2 alone.

Volunteers were 59 University students and staff without prior driving experience using the system. They were separated in to two groups (Alpha \& Beta) and sub-divided further between four courses used for testing so that there were about eight drivers in each sub-group. Drivers were shown the vehicle route and the obstacles placed on the route. Then each driver carried out a driving task ten times with sensors to assist them and then ten times without any sensors. Then, groups repeated each test but this time using a new setting. A second group of tests examined the way that drivers drove through a route when confronted with new conditions after they had already developed their vehicle driving skill.

Subjective information about preference was also collected at the end of the testing using a simple questionnaire. Questions were:

"Do you prefer Setting 0 or Setting 3 support?"

"Is it easier to drive with Setting 0 or Setting 3?"
Answers are recorded in Table I.

TABLE I. ANSWERS FROM THE QUESTIONNAIRE

\begin{tabular}{|l|l|l|l|l|l|}
\hline & $\begin{array}{l}\text { Strongly } \\
\text { prefer 0 }\end{array}$ & $\begin{array}{l}\text { Prefer } \\
\text { 0 }\end{array}$ & Undecided & $\begin{array}{l}\text { Prefer } \\
3\end{array}$ & $\begin{array}{l}\text { Strongly } \\
\text { prefer 3 }\end{array}$ \\
\hline Preference & 12 & 14 & 19 & 10 & 4 \\
\hline $\begin{array}{l}\text { How } \\
\text { Easy? }\end{array}$ & 15 & 18 & 7 & 12 & 7 \\
\hline
\end{tabular}

\section{B. Results from Initial Testing}

Average completion times in seconds for Alpha and Beta for each attempt are shown in Fig. 1 and 2 for the four courses.

Tests 1 to 10 correspond to the initial tests (Alpha with Setting 3 support and Beta with Setting 0) and 11 to 20 show the later tests (Beta with Setting 3 support and Alpha with Setting 0).

Fig. 1 and 2 shows that Drivers in Alpha (with forcefeedback applied) learnt in a smoother manner and more quickly than Group Beta. This implies a supportive effect when using Setting 3 at first and while early learning is taking place and skills are being developed. In addition, the drivers in Group Alpha performed better in the second part of the tests, with assistance set at Setting 0. The acquisition of new skills was faster when using Setting 3 (a combination of both Setting 1 and Setting 2).

Drivers may have reached a general skill level and they were able to apply that skill to new conditions. In this case the new condition was to drive the vehicle without any assistance. Drivers in Beta did not appear to improve any further during the second half of testing. The time taken and the stability of the results were similar. That could be because the new skills that were gained using Setting 0 assistance does not easily transfer to new conditions and situations.

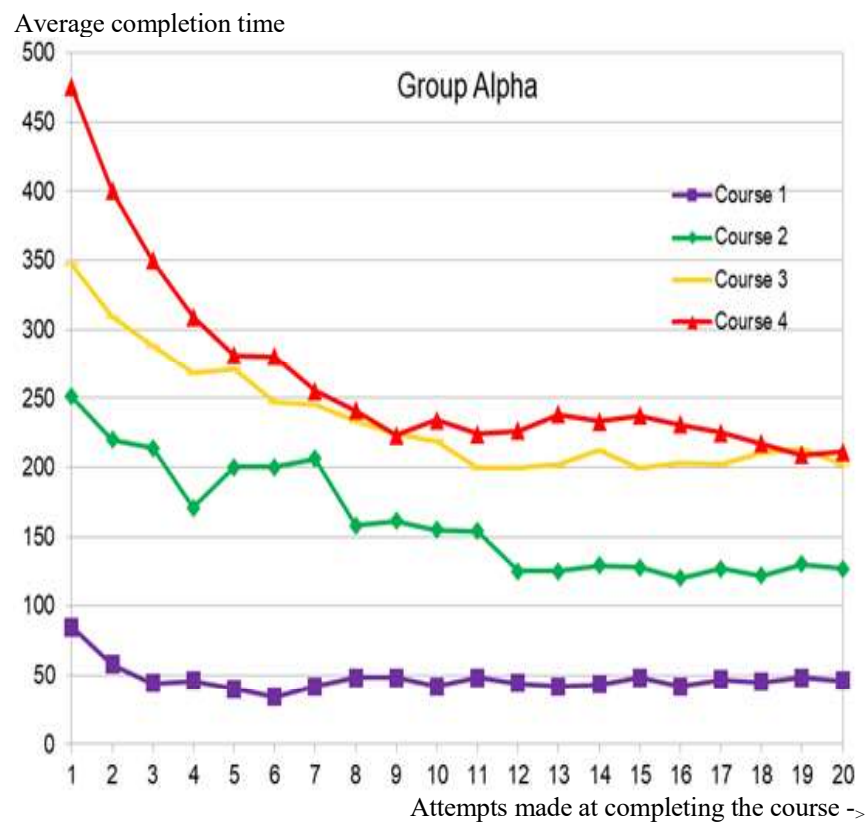

Fig. 1. Average completion time for Groups Alpha and Beta over four dissimilar courses. Setting 3 support first, and then Setting 0. 


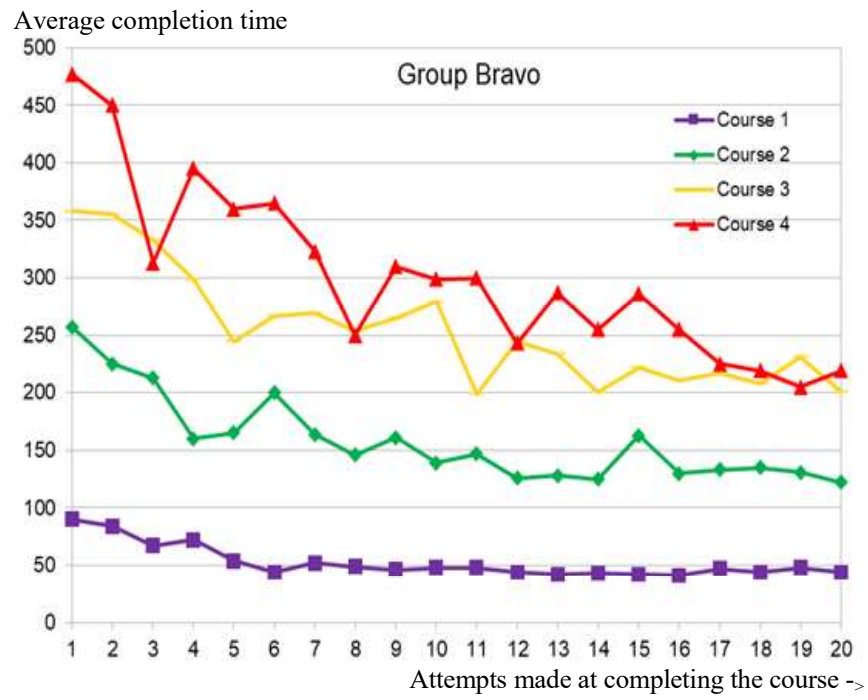

Fig. 2. Average completion time for Groups Alpha and Beta over $4 \mathrm{x}$ dissimilar courses. Setting 0 first and then Setting 3.

Table I shows the results from subjective appraisal (acquired from the questionnaires). Drivers said it was simpler to drive without any assistance from the sensor systems (Setting 0) but that disagreed with the more objective results. In addition, the questionnaire answers suggested that participants did not like being assisted.

\section{Discussion of Initial Testing}

The initial tests suggested vehicle drivers trained with sensors helping them could deal with new situations when they were not being assisted. That is when the sensors were switched off or removed.

Having some support from sensors during the learning phases appears to have had a significant effect on future working when participants completed driving tasks without any sensor assistance.

It is possible that participants may have developed an understanding of the systems and of driving the vehicle along a path during sequences of tests. More tests are needed to validate the results and check a driving skill can transfer across to a new working condition; one lacking any assistance from sensors.

Results do agree with results presented by Chikura [17] but the performance of participants faced with different tasks must be examined further to confirm and generalize the results.

The data collected suggests that vehicle drivers trained without any assistance from sensors did not function any better than drivers being assisted by sensors.

Results also indicated that participants that were taught without any assistance did not appear to learn steadily when compared with participants without previous experience. It is possible that skills obtained without sensor assistance had adverse effects on driving if assistance was added afterwards. That result is significant for and should be considered when establishing training processes for intelligent systems.

Off-the-record conversation suggested that this was partially because drivers thought their freedom to make decisions and to make move as they wished was hampered. That contradicted the more objective results obtained from the recording the average completion time for a driving task. That suggested that self-biased observations and criticisms should not be used to make decisions about training. Considering student preference appears to lead to inferior application.

\section{Second Set of Tests}

The second set of tests investigated the way that participants adapted their conduct if work conditions changed. Drivers used different sensor settings to provide different amounts of driving assistance. A different route was utilized for these tests that were more complicated and longer. Obstacles could be moved to easily create three discrete (but related) test runs using the same general route.

The second set of tests were to investigate differences in the behaviour of the participants if different levels of sensor support were provided and working conditions were changed.

Driving routes were carried out six times. Then routes were modified by moving obstacles and the participants drove the second route six times. Then routes were modified again by moving obstacles to new positions for a third set of six tests.

Each participant completed each test with the same level settings for each of their attempts.

Twelve new participants were separated into groups: Group Phi $\Phi$, Group Chi, X and Group Psi $\Psi$. $\Phi$ undertook tasks at Setting 0 (without assistance), $X$ at Setting 1 (sensors generating a repulsion force) and $\Psi$ at Setting 2 (automatic steering around obstacles).

The number of collisions and the time taken were logged.

\section{E. Results from the Second Set of Tests}

Fig. 3 to 5 shows the time taken in seconds for Groups $\Phi$, $\mathrm{X}$ and $\Psi$.

Setting 3 supports resulted in the fewest collisions but completion times became worse compared with Setting 0 support.

Whenever the course layout was changed (position of obstacles, etc.) then the differences in completion time was noted. Fig. 3 is smoother than Fig. 4 or Fig. 5 because completion time increased for Setting 1 and Setting 2, but did not change much for Setting 0 .

This suggested that skills learned without any driver assistance might be more useful than skills learned with driver assistance provided. 


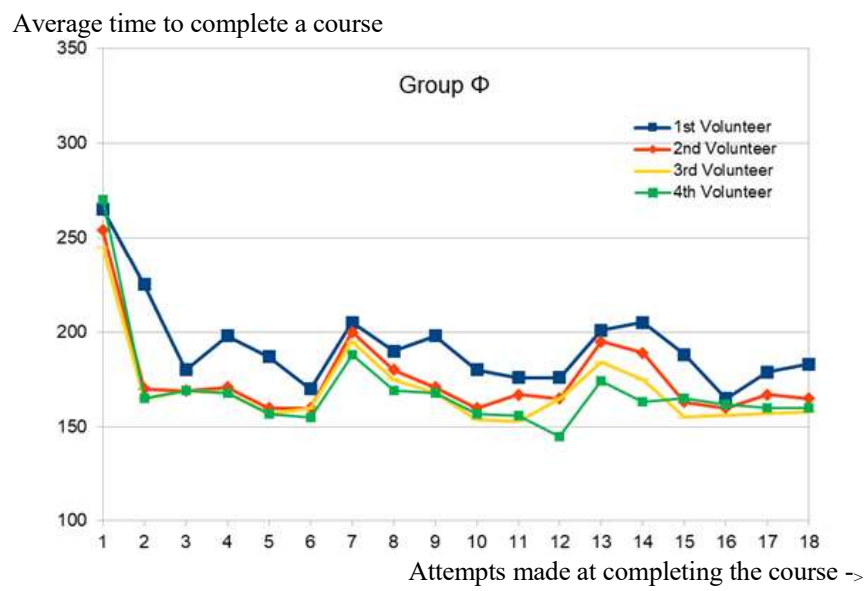

Fig. 3. Setting $0-$ No driver assistance.

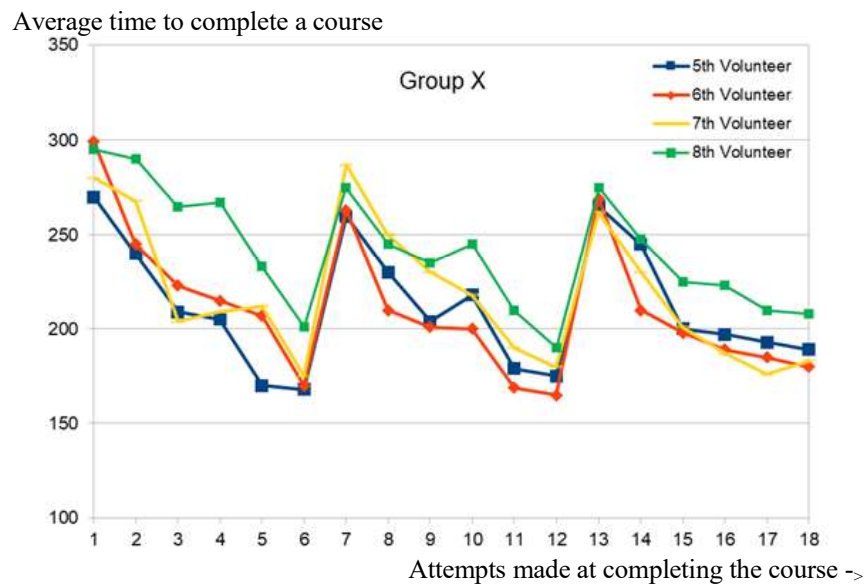

Fig. 4. Setting 1 - Ultrasonic sensors providing a repulsive force.

Fig. 6 shows the number of collisions for each driver. There appeared to be variances in driver behaviour and adaptation when the levels of assistance were changed and when participants come upon different working conditions. Average completion time changed for each setting whenever a course was altered.

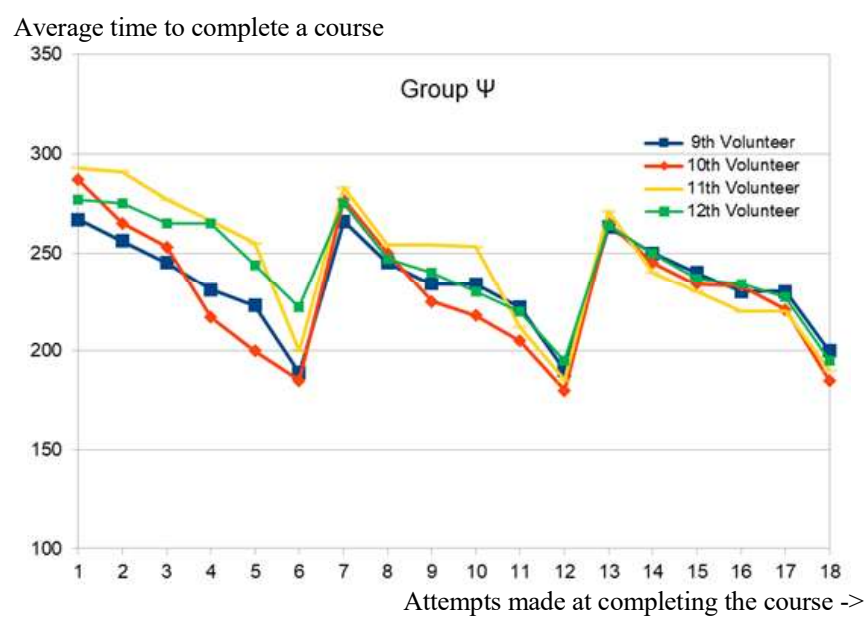

Fig. 5. Setting 2 - System automatically steering away from obstacles.

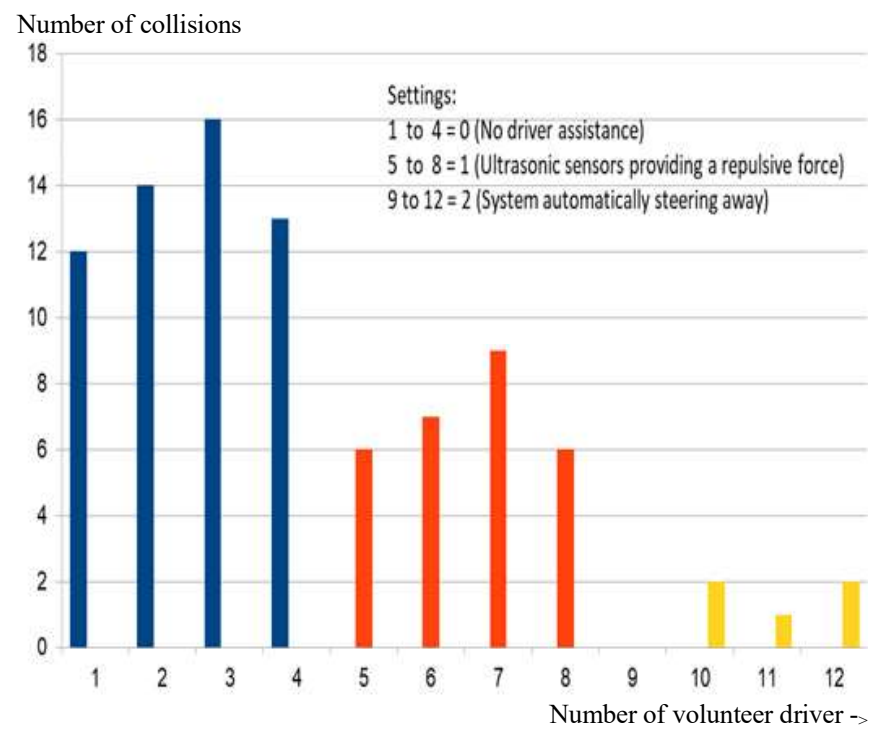

Fig. 6. Number of collisions for each vehicle driver.

\section{DISCUSSION AND CONCLUSIONS}

The way that drivers adapted their behaviour was studied when different levels of assistance were provided to decide if using sensors to assist during learning had any effect, and if so then what effect did they have?

A positive effect was observed when learning with assistance from sensors. A harmful effect was observed when learning without any assistance from the sensors but then using sensors later to assist participants with their driving. Driving at Setting 2 decreased the number of collisions in a variety of situations and arrangements of obstacles. The second set of tests contradicted some results obtained during initial tests and a larger number of participants could have made the results more significant.

The first set of tests suggested that support was helpful during learning but that was not seen in the second set of results. More tests are needed to focus on individual performance to acquire more unambiguous results. That said, the results have offered some insights about driver behaviour when learning with different levels of assistance provided by sensor systems. It may also suggest that drivers who learnt without sensors might find it harder to navigate when presented with extra information from sensors

This work suggests that using sensors during training is effective but some research has suggested that if a driver is proficient then they function better without help in open and safe conditions. In all situations though, assistance becomes more useful as environments become more complicated. Work is now examining the mixing of AI tools [21]-[42] to make use of particular AI tools where they are most useful.

\section{REFERENCES}

[1] D.A. Sanders, Ndzi, D, Chester, S \& Malik, M 2017, Adjustment of teleoperator learning when provided with different levels of sensor support while driving mobile robots. in $\mathrm{Y} \mathrm{Bi}$, S Kapoor \& $\mathrm{R}$ Bhatia (eds), SAI Intelligent Systems (IntelliSys) vol. 2, Lecture Notes in Networks and Systems, vol. 16, Springer, UK, pp. 548-558. DOI: 10.1007/978-3-31956991-8_41. 2017. 
[2] D.A. Sanders, Sanders, H, Gegov, A \& Ndzi, D 2017, Rule-based system to assist a tele-operator with driving a mobile robot. in $\mathrm{Y} \mathrm{Bi}, \mathrm{S}$ Kapoor \& R Bhatia (eds), SAI Intelligent Systems (IntelliSys) vol. 2, Lecture Notes in Networks and Systems, vol. 16, Springer, pp. 599-615, DOI: 10.1007\%2F978-3-319-56991-8 44. 2017.

[3] D.A. Sanders, 'Using self-reliance factors to decide how to share control between human powered wheelchair drivers and ultrasonic sensors' IEEE Transactions on Neural Systems and Rehabilitation Engineering, vol 25, no. 8, pp. 1221-1229. DOI: 10.1109/TNSRE.2016.2620988. 2017.

[4] D.A. Sanders, 'Non-model-based control of a wheeled vehicle pulling two trailers to provide early powered mobility and driving experiences' IEEE Transactions on Neural Systems and Rehabilitation Engineering. Vol 26 (1), pp: 96 - 104. DOI: 10.1109/TNSRE.2017.2726443. 2018.

[5] D.A. Sanders, Gegov, A, Tewkesbury, G \& Khusainov, R, Rule-based system to assist a powered wheelchair driver. IEEE Proceedings of the Intelligent Systems Conference (IntelliSys) 2017. IEEE, London, United Kingdom, 7-8 September. ISBN 978-1-5090-6435-9, pp: 558-565. 2017.

[6] D.A. Sanders, Sanders, B, Gegov, A \& Ndzi, D, Results from investigating powered wheelchair users learning to drive with varying levels of sensor support. in Proceedings of the Intelligent Systems Conference (IntelliSys) 2017. IEEE, London, United Kingdom, 7-8 September. ISBN 978-1-5090-6435-9, pp: 241-245. 2017.

[7] D.A. Sanders, Sanders, B, Ndzi, D \& Bausch, N, Using confidence factors to share control between a mobile robot tele-operater and ultrasonic sensors. in Proceedings of SAI Intelligent Systems Conference (IntelliSys) 2017. IEEE, London, United Kingdom, 7-8 September. ISBN 978-1-5090-6435-9, pp: 1026-1033. 2017,

[8] D.A. Sanders, Gegov, A \& Ndzi, D, Knowledge-based expert system using a set of rules to assist a tele-operated mobile robot. in $\mathrm{Y} \mathrm{Bi}, \mathrm{S}$ Kapoor \& R Bhatia (eds), Intelligent Systems and Applications. Studies in Computational Intelligence, vol. 751, Springer, pp. 371-392. 2017.

[9] US: Mobileye intros smartphone connected driver assistance (ADAS) technology". Telematics News 2012. http://telematicsnews.info/2012/01/12/us-mobileye-intros-smartphoneconnected-driver-assistance-adas-technology j3122. Retrieved $13 / 11 / 2017$.

[10] I. Riches. "Strategy Analytics: Automotive Ethernet: Market Growth Outlook | Keynote Speech 2014 IEEE SA: Ethernet \& IP @ Automotive Technology Day" (PDF). http://standards.ieee.org/events/automotive/2014/00_Automotive_Ethern et_Market_Growth_Outlook.pdf. Retrieved 13/11/2017.

[11] K. Rees and J S Park, Vehicle Information Access API". W3C Business Group report, W3C and LG Electronics. 2014 https://www.w3.org/2014/automotive/vehicle_spec.html.

Retrieved 13/11/2017.

[12] ADAS Definition". Autoconnectedcar.com. Archived from the original on $06 / 10 / 2012$ https://web.archive.org/web/20120610055853/http://telematicsnews.info /2012/01/12/us-mobileye-intros-smartphone-connected-driverassistance-adas-technology_j3122. Retrieved 13/11/2017.

[13] Banga, Bhavya, Global ADAS and Autonomous Driving Components Market, Analysis \& Forecast. Business Intelligence and Strategy Research. 2017. https://bisresearch.com/industry-report/global-adasautonomous-driving-components-market-2026.html.

Retrieved 13/11/2017

[14] Sanders DA; Graham-Jones J and Gegov A. Improving ability of teledrivers to complete progressively more difficult mobile robot paths using simple expert systems and ultrasonic sensors. Industrial Robot Volume: 37, Issue: 5, Pages: 431-440. 2010

[15] Sanders, D. A., Bausch, N. C. \& Ndzi, D. L Changes to user learning behaviour of vehicle drivers depending on the level of sensor support. IEEE Proc' of International Conference Health Informatics and Medical Systems HIMS'15. Arabnia, H. R. \& Deligiannidis, L. (eds.). Published by CSREA Press, pp: 62-65. 2015.

[16] Sanders DA, Stott I, Robinson D and Ndzi D. Analysis of successes and failures with a tele-operated mobile robot in various modes of operation. Robotica 30, pp 973-988. 2012.

[17] D Chikura, M Takahashi, S Watanabe and M Kitamura, Adaptation of
User Behavior to the Different Level of Tele-Operation Support:. IEEE International Conference on Systems, Man, and Cybernetics. Volume 3, pp: 739 - 744. 1999.

[18] Backes PG, "Supervised Autonomy for Space Robotics," Progress in Astronautics and Aeronautics 161, pp. 139-158. 1994.

[19] Draper JV et.al. "Measuring Operator Skill and Teleoprator Performance," Proc. of International Symposium on Teleoperation and Contro1, 1998.

[20] Volpe R, "Techniques for Collision Prevention ,Impact Stability, and Force Control by Space Robots", Progress in Astronautics and Aeronautics, Vol.161, 1994, pp.I75-212.

[21] Gegov A, Arabikhan F, Sanders D, Vatchova B and Vasileva, T "Fuzzy networks with feedback rule bases for complex systems modelling". International Journal of Knowledge-Based and Intelligent Engineering Systems, vol 21, no. 4, pp. 211-225.. 2017.

[22] Gegov A, Petrov, N, Sanders D and Vatchova B, "Boolean matrix equations for node identification in fuzzy rule based networks", International Journal of Knowledge-Based and Intelligent Engineering Systems. 21, 2, p. 69-83. 2017

[23] Gegov A, Petrov, N, Sanders D and Vatchova, B. "Modular rule base fuzzy networks for linguistic composition based modelling", International Journal of Knowledge-Based and Intelligent Engineering Systems. 21, 2, p. 53-67. 2017.

[24] Gegov A, Sanders D and Vatchova, B "Aggregation of inconsistent rules for fuzzy rule base simplification", International Journal of KnowledgeBased and Intelligent Engineering Systems. Knowledge-Based and Intelligent Engineering Systems, vol 21, no. 3, pp. 135-145. DOI: 10.3233/KES-170358. 2017.

[25] Mohd-Safar, NZ, Ndzi, D, Sanders, D, Noor, HM \& Kamarudin, LM 2018, Integration of Fuzzy C-Means and artificial neural network for short-term localized rainfall forecasting in tropical climate. $\mathrm{Y} \mathrm{Bi}, \mathrm{S}$ Kapoor \& R Bhatia (eds), Studies in Computational Intelligence, vol. 751, Springer, pp. 325-348. 2018.

[26] Sanders, D 2017, 'New method to design large scale high-recirculation airlift reactors', Proc of Inst of Civil Eng; Journal of Environmental Engineering and Science, vol 12, no. 3, pp. 62-78. 2017.

[27] Sanders, DA, Bergasa-Suso, J, Khusainov, R, Gegov, AE, Chester, SD \& Bausch, NC 2016, Introducing dead bands within two-dimensional clusters of user data to improve data classification. Proceedings of the 9th International Conference on Human Systems Interaction (HSI). IEEE, pp. 14-20, 9th International Conference on Human System Interactions, UK, 6/07/16. 2016.

[28] Sanders, DA, Langner, M, Gegov, AE, Ndzi, DL, Sanders, H \& Tewkesbury, GE "Tele-operator performance and their perception of system time lags when completing mobile robot tasks". Proceedings of the 9th International Conference on Human Systems Interaction (HSI). IEEE, pp. 236-242, 9th International Conference on Human System Interactions, UK, 6/07/16. 2016.

[29] Gegov, AE, Sanders, DA \& Vatchova, B "Mamdani fuzzy networks with feedforward rule bases for complex systems modelling" Journal of Intelligent \& Fuzzy Systems, vol 30, no. 5, pp. 2623-2637. 2016.

[30] Robinson, DC, Sanders, DA \& Mazharsolook, E, "Ambient intelligence for optimal manufacturing and energy efficiency" Assembly Automation, vol 35, no. 3, pp. 234-248. 2016.

[31] Gegov, A, Sanders, D \& Vatchova, B "Complexity management methodology for fuzzy systems with feedforward rule bases". International Journal of Knowledge-Based and Intelligent Engineering Systems, vol 19, 310, pp. 83-95. 2016.

[32] Sanders, DA \& Bausch, NC "Improving steering of a powered wheelchair using an expert system to interpret hand tremor". in H Liu, N Kubota, X Zhu, R Dillmann \& D Zhou (eds), Intelligent robotics and applications: Part II. Lecture notes in artificial intelligence, vol. 9245, Springer, Switzerland, pp. 460-471. 2015.

[33] Robinson, DC, Sanders, DA \& Mazharsolook, E "Ambient intelligence for optimal manufacturing and energy efficiency" Assembly Automation, vol 35, no. 3, pp. 234-248. DOI: 10.1108/AA-11-2014-087. 2015.

[34] Gegov, A, Sanders, D \& Vatchova, B "Complexity management methodology for fuzzy systems with feedforward rule bases" 
International Journal of Knowledge-Based and Intelligent Engineering Systems, vol 19, 310, pp. 83-95. DOI: 10.3233/KES-150310. 2015.

[35] Sanders, DA, Tewkesbury, GE \& Gegov, AE "Fast transformations to provide simple geometric models of moving objects". in H Liu, $\mathrm{N}$ Kubota, X Zhu, R Dillmann \& D Zhou (eds), Intelligent robotics and applications Part I. Lecture notes in computer science, vol. 9244, Springer, pp. 604-617. DOI: 10.1007/978-3-319-22879-2_55. 2015.

[36] Gegov, A, Arabikhan, F \& Sanders, D "Rule base simplification in fuzzy systems by aggregation of inconsistent rules" Journal of Intelligent \& Fuzzy Systems, vol 28, no. 3, IFS1418, pp. IFS1418. DOI: 10.3233/IFS-141418. 2015.

[37] Gegov, A, Sanders, D \& Vatchova, B "Complexity management methodology for fuzzy systems with feedback rule bases" Journal of Intelligent \& Fuzzy Systems, vol 26, no. 1, pp. 451-464. DOI: 10.3233/IFS-130857. 2014.

[38] D.A. Sanders, A. Gegov, M. Haddad, F. Ikwan, D. Wiltshire and Y. C. Tan "A Rule-based Expert System to decide on direction and speed of a powered wheelchair". IEEE Proceedings of the SAI Conference on Intelligent Systems 2018 (In Press).
[39] D. Sanders, A. Gegov, G. E. Tewkesbury and R. Khusainov "Sharing driving between a vehicle driver and.a sensor system using trust-factors to set control gains. IEEE Proceedings of the SAI Conference on Intelligent Systems 2018 (In Press).

[40] M. Haddad, D. A. Sanders, N. Bausch, G. Tewkesbury, A. Gegov and M. Hassan, "Learning to make intelligent decisions using PROMITHEE II and the Analytical Hierarchy Process". IEEE Proceedings of the SAI Conference on Intelligent Systems 2018 (In Press).

[41] D. Sanders, D. C. Robinson, M. Hassan, M. Haddad, A. Gegov and N. Ahmed "Making decisions about saving energy in Compressed Air Systems using Ambient Intelligence and AI". IEEE Proceedings of the SAI Conference on Intelligent Systems 2018 (In Press).

[42] D. Sanders, Q. Wang, N. Bausch, Y. Huang, S. Khaustov and I. Popov "An efficient method to produce minimal real time geometric representations of moving obstacles". IEEE Proceedings of the SAI Conference on Intelligent Systems 2018 (In Press). 